\title{
Short communication: A competitive exclusion study reveals the emergence of Bacillus subtilis as a predominant constitutive microorganism of a whey reverse osmosis membrane biofilm matrix
}

\author{
Pratishtha Verma, Neha Singh, and Sanjeev Anand* ${ }^{*}$ \\ Midwest Dairy Foods Research Center, Dairy and Food Science Department, South Dakota State University, Brookings 57007
}

\begin{abstract}
Microbial attachment and colonization on separation membranes lead to biofilm formation. Some isolates within the biofilm microflora acquire greater resistance to the chemical cleaning protocols on prolonged use of membranes. It is thus likely that the constitutive microflora might compete with each other and result in certain species emerging as predominant, especially within older biofilms. To understand the microbial interactions within biofilms, the emergence of predominance was studied in the current investigation. An 18-mo-old reverse osmosis membrane was procured from a whey processing plant. The membrane pieces $(2.54 \times 2.54$ $\mathrm{cm}^{2}$ ) were neutralized by dipping in Letheen broth. The resuscitation step was done in tryptic soy broth (TSB) at $37^{\circ} \mathrm{C}$, followed by plating on tryptic soy agar (TSA) to recover the constitutive microflora. Distinct colonies of isolates were further identified using MALDI-TOF as Bacillus licheniformis, Exiguobacterium aurantiacum, Acinetobacter radioresistens, Bacillus subtilis (rpoB sequencing), and 1 unidentified species each of Exiguobacterium and Bacillus. Further, the competitive exclusion study helped establish the emergence of predominance using a co-culturing technique. Fifteen combinations (of 2 isolates each) were prepared from the isolates. Pure cultures of the respective isolates were spiked in a ratio of $1: 1$ in TSB and incubated at $37^{\circ} \mathrm{C}$ for 24 $\mathrm{h}$, followed by plating on TSA. The enumerated colonies were distinguished based on colony morphology, Gram staining, and MALDI-TOF to identify the type of the isolate. Plate counts of B. subtilis emerged as predominant with mean log counts of $7.22 \pm 0.22 \mathrm{cfu} /$ $\mathrm{mL}$. The predominance of $B$. subtilis was also validated using the process of natural selection in a multispecies growth environment. In this instance, the TSB culture with overnight-incubated membrane piece (with mixedspecies biofilm) at $37^{\circ} \mathrm{C}$ for $12 \mathrm{~h}$ was inoculated in fresh
\end{abstract}

Received March 5, 2020.

Accepted August 7, 2020.

*Corresponding author: Sanjeev.anand@sdstate.edu
TSB and incubated for the second cycle. Overall, 5 such sequential broth-culture incubation cycles were carried out, followed by pour plating on TSA plates, at the end of each cycle. The isolates obtained were identified using a similar methodology as mentioned above. The fifth subsequent transfer depicted the presence of only 1 B. subtilis isolate on plating, thereby validating its predominance under the conditions of the experiment. Key words: co-culture combination, natural selection, subsequent transfer

\section{Short Communication}

Separation membranes are now increasingly used in the dairy and food processing industry (Anand et al., 2012) for diverse applications such as whey protein concentration, milk protein standardization, desalination, wastewater treatment, and so on (Daufin et al., 2001; Turan et al., 2002). Despite numerous benefits, membrane processing has a major limitation of biofouling due to bacterial biofilm formation on the membrane surfaces (Suwarno et al., 2012). Multispecies biofilms are formed when feed containing microorganisms comes in contact and initiates colonization on the membrane surface (Anand et al., 2012). The multispecies biofilms are known to be more complex and tend to form thicker and more stable biofilms, which could be due to the release of extracellular polymeric substances (EPS) by various bacterial species (Hassan et al., 2010; Anand et al., 2014). The EPS secreted by multiple species in a biofilm matrix acts as a shield and helps develop resilient biofilms that are very difficult to clean (Anand et al., 2014). Even the cleaning and sanitization protocols prove to be ineffective for removing such resilient biofilms. Such biofouling not only results in a reduced flux rate but also serves as a constant source of contamination, hence leading to premature replacement of separation membranes and economic losses faced by the dairy industry (Marchand et al., 2012). The membrane replacement cost is high and could constitute around 25 to $40 \%$ of the cost of the membrane plant (D'Souza and Mawson, 2005). Previous studies have reported the 
presence of Enterococcus, Staphylococcus, Micrococcus, Streptomyces, Corynebacterium, Bacillus, Klebsiella, Aeromonas, Pseudomonas, Escherichia coli, Streptococcus, Chryseobacterium, and bacteria from Firmicutes and Proteobacteria phylum as constitutive microflora found on dairy separation membranes (Avadhanula, 2011; Chamberland et al., 2017b).

Some organisms present within the biofilm microflora show predominance over others over the prolonged use of membranes (Anand and Singh, 2013). Some previous studies have also depicted the predominance of single species in biofilms in different dairy processes (Flint et al., 1997; Nadell et al., 2016). Competition among microorganisms in a shared environment occurs in all but the simplest ecosystems (Fredrickson and Stephanopoulos, 2017). When competition is focused on a single resource in a common setting, one microbial population outcompetes the other, hence emerging as predominant in a constitutive microflora. Several factors can be responsible for the emergence of predominance, such as competition for the nutrients, faster-growing rate of one microorganism over the others, production of metabolites by the cells, production of bacteriocin, secretion of broad- and narrow-spectrum toxins with coupled privatized antitoxin, and so on (Weber et al., 2014; Nadell et al., 2016; Frost et al., 2018). Competitive exclusion is a unique interaction between species that leads to predominance along with various degrees of coexistence (DeBach, 1966; Weiner et al., 2017). The coexistence of all strains depends mainly on 3 factors: the relative growth rate of the microorganisms present in a mixed species, a ratio of the inoculum, and an effective range of toxins (Weber et al., 2014). Studies also support that coexistence is eventually lost as a result of selective sweeps in growing microbial colonies (Korolev et al., 2012). Therefore, it is important to understand the emergence of predominance within membrane biofilms. This could provide vital information on the development of mature biofilms on prolonged use of membranes and the potential for selective species to generate resistances leading to the predominance of one microorganism over others within a biofilm matrix. This information would be useful to create targeted clean-in-place (CIP) strategies to eliminate the resistant biofilm microflora and extend the service life of filtration membranes in the dairy industry in addition to reducing cross-contamination.

An 18-mo-old reverse osmosis whey concentration membrane was procured from a whey processing commercial plant located in the Midwest region of the United States. The used membrane was drawn after the completion of the regular CIP cycle. The CIP protocol involved a 5-wash-step process with an intermittent
Table 1. Cleaning cycles with clean-in-place time and temperatures

\begin{tabular}{lrc}
\hline Step & Time $(\min )$ & Temperature $\left({ }^{\circ} \mathrm{C}\right)$ \\
\hline Flush 1 & 9 & \\
Wash 1 & 35 & 46.11 \\
Flush 2 & 9 & 46.11 \\
Wash 2 & 35 & \\
Flush 3 & 9 & 46.11 \\
Wash 3 & 40 & 23.88 \\
Flush 4 & 9 & \\
Wash 4 & 0 & 23.88 \\
Flush 5 & 0 & \\
Wash 5 & 5 & \\
Flush 6 & 5 & \\
\hline
\end{tabular}

${ }^{1}$ Flushes 1 to 6 refer to caustic flush. Wash 1,2,3,4, and 5 refer to caustic wash, acid wash, enzyme wash, caustic wash 2 , and sanitizer wash, respectively.

caustic flush at the end of each wash step. A typical cycle included caustic wash, acid wash, enzyme wash, caustic wash 2 , followed by a sanitizer wash, per the recommendations of the manufacturer (Table 1). The whey filtration process in that plant was a continuous run used to filter 350 gallons of cheese whey/min at about 15 to $19^{\circ} \mathrm{C}$. The routine CIP protocol involved the combination of alkaline, acid, and enzyme wash at the end of each cycle. The membrane was sealed aseptically in a plastic wrap before bringing it to our laboratory for analysis purposes.

The constitutive microflora was isolated following the protocol described by Anand and Singh (2013) with slight modifications. A cross-sectional piece was aseptically cut out from an 18-mo-old membrane with the help of a reciprocating saw (DWE 304, Dewalt Industrial Tool Co., Towson, MD). Aseptic conditions were maintained by sterilizing the blade of the electric saw and wiping the surrounding area with $70 \%$ ethanol before cutting the membrane. Six membrane pieces $(2.54$ $\times 2.54-\mathrm{cm}^{2}$ ) were cut with a pair of sterile scissors, and any chemical residue on the membrane pieces was neutralized using Letheen broth (Puritan ESK sampling kits with pre-filled Letheen broth, Puritan, Guilford, $\mathrm{ME})$. The composition of Letheen broth is designed to support the growth of a variety of microorganisms, while lecithin helps neutralize quaternary ammonium compounds, and the presence of Tween 80 enables it to neutralize the phenolic disinfectants and hexachlorophene present in the disinfectant; together, they neutralize ethanol (Hardy Diagnostics, 2020). Three neutralized membrane pieces were resuscitated by suspending each membrane piece in a bottle containing $200 \mathrm{~mL}$ of tryptic soy broth (TSB; Bacto, Rockville, $\mathrm{MD}$ ) and incubating for $12 \mathrm{~h}$ at $37^{\circ} \mathrm{C}$. It is also a common incubation temperature used for resuscitation of microorganisms with a variable time length. The TSB, 
being a nonspecific medium, was used for the resuscitation process to provide a suitable environment for a variety of the stressed intact microorganisms to grow (Stephens and Mackey, 2003). After resuscitation, the bottles were taken out from the incubator and the broth was properly mixed by shaking the bottle containing the suspended membrane piece 25 times in a 1-foot arc within $7 \mathrm{~s}$ before plating (US FDA, 1969). Serial dilutions (up to $10^{-7}$ ) were pour plated on tryptic soy agar (TSA) and incubated at $37^{\circ} \mathrm{C}$ for $24 \mathrm{~h}$ to enumerate the constitutive microflora. The membrane used for separation operated under conditions close to mesophilic growth (the feed for the reverse osmosis membrane was in the range of $15-19^{\circ} \mathrm{C}$ ), which would mostly support the growth of mesophilic organisms in the biofilm matrix. Distinct colonies obtained on the plates prepared via enrichment process were distinguished based on colony morphology and Gram staining. Based on the similarity in colony morphology, multiple colonies were picked up (2-5 of each type of colony morphology) and were sent out for MALDI-TOF identification at the Veterinary Science Department, South Dakota State University, Brookings. The information was consolidated and presented. The MALDI-TOF resulted in the identification of most of the isolates except 3 isolates, which were identified only to the genus level. Out of these, 1 Bacillus isolate, later also identified as the predominant species of the constitutive microflora, was identified up to its species level using rpoB sequencing (Food Science, Cornell University, Ithaca, NY; GenBank accession no. MT650127).

The competitive exclusion study helped establish predominance using the coculture technique. Six isolates were identified based on colony morphology and Gram staining followed by MALDI-TOF identification and were named VQ1, VQ2, VQ3, VQ4, VQ5, and VQ6. The list of the isolates is presented in Figure 1. All isolates were individually grown in TSB at $37^{\circ} \mathrm{C}$ for 24 $\mathrm{h}$, and the plate count exhibited an average mean count of $7 \log \mathrm{cfu} / \mathrm{mL}$. For the competitive exclusion studies, using the coculture technique, 6 isolates were paired into 15 possible combinations of 2 isolates each (Table 2 ). For each of these combinations, the overnight-grown isolates were suspended in a 1:1 ratio in TSB, and the cocultures were incubated at $37^{\circ} \mathrm{C}$ for $24 \mathrm{~h}$. Serial dilutions were prepared, and plating was carried out on TSA. As explained before, the isolates on the plates were distinguished and identified. Finally, the plate counts were compared to establish the predominance of one culture over the other in the coculture growth.

The results of the coculture growth were further validated using the process of natural selection in a multispecies growth environment. For the natural selection study, a $2.54 \times 2.54-\mathrm{cm}^{2}$ piece of 18 -mo-old reverse osmosis membrane was suspended in Letheen broth to neutralize any chemical residue, and then the membrane piece was suspended in TSB and incubated at $37^{\circ} \mathrm{C}$ for $12 \mathrm{~h}$ (cycle 1 incubation). The subculturing of the broth was followed by 5 cycles of subsequent transfers (Korolev et al., 2012). Simultaneously, serial dilutions were prepared at the end of each cycle and plated on TSA using the pour plate technique. The enumerated organisms were identified. The flowchart of the experimental design is shown in Figure 2. All the experiments were carried out in duplicate, and the data were calculated for means values and standard deviations. The culturing techniques used to isolate constitutive microflora revealed the presence of grampositive as well as gram-negative bacteria. The distinct isolates obtained using the enrichment process under the conditions of experimentation were distinguished based on colony morphology and Gram staining. Individually streaked distinct isolates were then outsourced for MALDI-TOF identification. From these, 6 isolates were identified as Bacillus licheniformis, Exiguobacterium aurantiacum, Acinetobacter radioresistens, 1 unidentified species of Exiguobacterium, and 2 species of Bacillus. Out of the unidentified species, 1 Bacillus strain depicting predominant characteristics (explained below in coculture experiment) was outsourced for rpoB sequencing and was identified as Bacillus subtilis. The other 2 isolates, which were identified at the genus level only, Exiguobacterium (VQ2) and Bacillus (VQ4), were different from the species identified from the constitutive microflora of the 18-mo-old membrane biofilm. Although the MALDI-TOF results did not exactly identify the species levels for these microorganisms (VQ2 and VQ4), they were differentiated from the rest of the isolates based on colony morphology and Gram staining. For the ease of working, these microorganisms were assigned isolate codes, which are depicted in Figure 1, along with their colony morphology and species name based on MALDI-TOF identification or rpoB sequencing. A previously validated protocol was used to isolate the biofilm microflora. In our previous studies, we successfully isolated biofilm microflora to recreate in vitro single and multispecies biofilms in our laboratory (Anand and Singh, 2013; Marka and Anand, 2018). A similar type of biofilm constitutive microflora was previously reported on spiral wound membranes, where species associated with Actinobacteria and Bacillus genera were observed (Chamberland et al., 2017b). Some other studies (Avadhanula, 2011; Tang et al., 2009) on whey reverse osmosis biofilms have reported the presence of Bacillus as part of biofilm microflora but did not report the presence of some other types of 


\begin{tabular}{|c|c|c|c|}
\hline $\begin{array}{l}\text { Isolate } \\
\text { code }\end{array}$ & $\begin{array}{l}\text { Description of } \\
\text { colony morphology }\end{array}$ & $\begin{array}{l}\text { Picture of colony } \\
\text { morphology }\end{array}$ & $\begin{array}{l}\text { MALDI-TOF identification/ } \\
\text { rpoB sequencing }\end{array}$ \\
\hline VQ1 & Irregular, undulated, and opaque & & Bacillus subtilis \\
\hline VQ2 & Round, raised, and translucent & & Exiguobacterium sp. \\
\hline VQ3 & Irregular, rough, and opaque & & Bacillus licheniformis \\
\hline VQ4 & Irregular, lobate, and opaque & & Bacillus sp. \\
\hline VQ5 & Round, entire, and opaque & & $\begin{array}{l}\text { Exiguobacterium } \\
\text { aurantiacum }\end{array}$ \\
\hline VQ6 & $\begin{array}{l}\text { Small colonies, round, and } \\
\text { opaque }\end{array}$ & & Acinetobacter radioresistens \\
\hline
\end{tabular}

Figure 1. Identification of constitutive microflora based on colony morphology and MALDI-TOF.

Table 2. Coculture combinations using 6 isolates identified from the constitutive microflora of an old reverse osmosis membrane

\begin{tabular}{|c|c|c|c|c|}
\hline \multicolumn{5}{|c|}{ Combination } \\
\hline $\begin{array}{l}\text { VQ1-VQ2 } \\
\text { VQ1-VQ3 } \\
\text { VQ1-VQ4 } \\
\text { VQ1-VQ5 } \\
\text { VQ1-VQ6 }\end{array}$ & $\begin{array}{l}\text { VQ2-VQ3 } \\
\text { VQ2-VQ4 } \\
\text { VQ2-VQ5 } \\
\text { VQ2-VQ6 }\end{array}$ & $\begin{array}{l}\text { VQ3-VQ4 } \\
\text { VQ3-VQ5 } \\
\text { VQ3-VQ6 }\end{array}$ & $\begin{array}{l}\text { VQ4-VQ5 } \\
\text { VQ4-VQ6 }\end{array}$ & VQ5-VQ6 \\
\hline
\end{tabular}




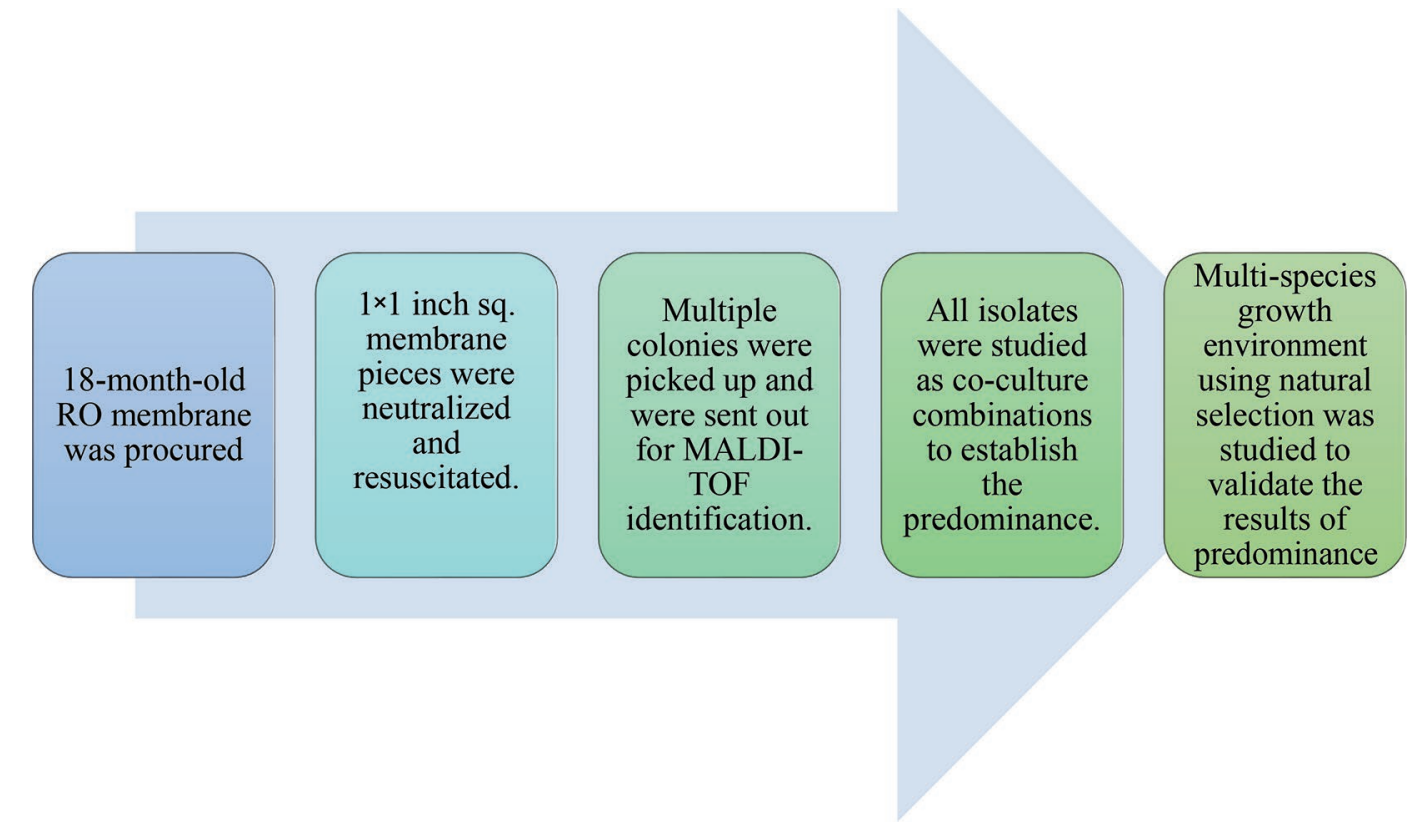

Figure 2. Flowchart of the experimental design followed to study the emergence of predominance within the constitutive microflora. RO $=$ reverse osmosis.

microflora found in the current study. This could be due to variations such as feed or operational parameters, as these factors are reported to greatly affect the diversity of bacteria colonizing membranes (Chamberland et al., 2017a).

To establish the emergence of predominance within the biofilm microflora of the 18-mo-old membrane used in our study, the interaction between 15 possible cocultures was investigated. All of the isolates were used to prepare combinations. Each coculture combination consisted of 2 isolates and expressed different colony morphology on TSA plates after the coculture interaction. The isolates obtained on the plates were identified using MALDI-TOF. The plate count of different isolates in the cocultures was recorded, and the $\log _{10}$ counts of each coculture combination were compared. Bacillus subtilis (VQ1) expressed itself in all the coculture combinations with a mean $\log$ count of 7.22 $\pm 0.22 \mathrm{cfu} / \mathrm{mL}$ (Table 3 ). In addition, it inhibited the growth of all the isolates in its combinations. It is interesting to note that another isolate, $B$. licheniformis was expressed equally in a coculture with $B$. subtilis and was not completely inhibited. From Table 3, it can be observed that for the coculture combinations VQ4 and VQ5, VQ5 and VQ6, and so on, the isolate that emerged as predominant was ultimately inhibited by VQ1 (B. subtilis) when grown under coculture growth conditions. Therefore, it can be inferred that B. subtilis emerged as predominant in a coculture combination growth environment. The data for all coculture com- binations are depicted in Table 3. Similarly, previous studies have reported the predominance of spore-forming Bacillus spp. in membrane biofilms (Avadhanula, 2011; Anand et al., 2014). This could be associated with commonly present gram-positive microorganisms or thermoresistant spore-forming Bacillus spp. in whey starter population (Tang et al., 2009). The presence of $B$. licheniformis alongside $B$. subtilis could be associated with the concept of coexistence (Borenstein et al.,

Table 3. Log counts $(\mathrm{cfu} / \mathrm{mL})$ of culture combinations after $24 \mathrm{~h}$ under coculture growth conditions

\begin{tabular}{lcc}
\hline $\begin{array}{l}\text { Coculture } \\
\text { combination }\end{array}$ & $\begin{array}{c}\text { First organism } \\
\text { in combination }\end{array}$ & $\begin{array}{c}\text { Second organism } \\
\text { in combination }\end{array}$ \\
\hline VQ1-VQ2 & $7.72 \pm 0.66$ & $7.49 \pm 1.14$ \\
VQ1-VQ3 & $6.83 \pm 0.67$ & - \\
VQ1-VQ4 & $7.23 \pm 0.25$ & - \\
VQ1-VQ5 & $7.53 \pm 0.59$ & - \\
VQ1-VQ6 & $7.27 \pm 0.48$ & $7.57 \pm 1.20$ \\
VQ2-VQ3 & $7.20 \pm 0.39$ & - \\
VQ2-VQ4 & $5.33 \pm 0.47$ & - \\
VQ2-VQ5 & $7.26 \pm 0.29$ & $6.94 \pm 0.35$ \\
VQ2-VQ6 & $6.75 \pm 0.55$ & - \\
VQ3-VQ4 & $7.30 \pm 1.16$ & - \\
VQ3-VQ5 & $7.69 \pm 1.32$ & - \\
VQ3-VQ6 & $7.81 \pm 1.27$ & $6.97 \pm 0.40$ \\
VQ4-VQ5 & $7.14 \pm 0.50$ & \\
VQ4-VQ6 & $7.30 \pm 0.32$ & \\
VQ5-VQ6 & - & \\
\hline
\end{tabular}

$\overline{{ }^{1} \mathrm{VQ1}=\text { Bacillus subtilis; VQ2 = Exiguobacterium } \mathrm{sp} \text {; VQ3 = Bacillus }}$ licheniformis; VQ4 = Bacillus sp.; VQ5 = Exiguobacterium aurantiacum; VQ6 = Acinetobacter radioresistens.

${ }^{2}$ Not detected. 


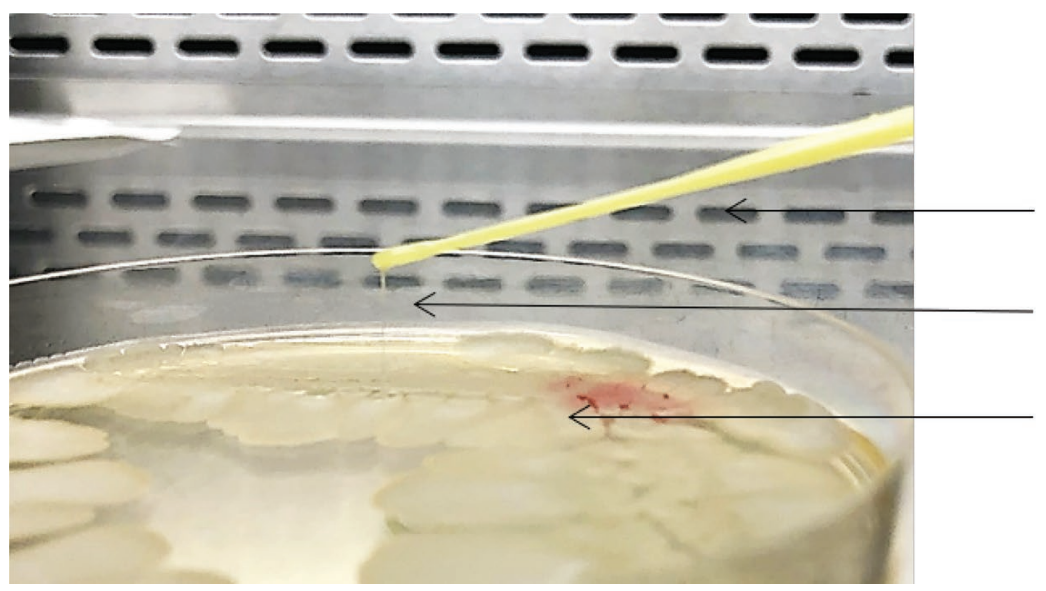

\section{Inoculation Loop}

String

Bacterial Growth

Figure 3. The string test was found to be positive for Bacillus subtilis, which had a hypermucoid appearance (string size $>5 \mathrm{~mm}$ ) when it formed a string.

2013; Abrudan et al., 2015), and both organisms have also been reported together in various raw milk samples (Brown, 2000; Buehner, 2014).

A string test was conducted on the colonies of $B$. subtilis and $B$. licheniformis isolates to compare the 2 isolates for their competing attributes in terms of EPS. The presence of mucoid colonies has been associated with the predominance of certain strains over others that do not produce mucoid colonies (Stratton, 1983; Chinen, 2017). The string test was found to be positive for B. subtilis only, and the string length was observed to be $>5 \mathrm{~mm}$ (Figure 3), demonstrating the hypermucoviscosity phenotype. This could also be one of the potential contributing factors for the predominance of $B$. subtilis in a coculture growth. In addition, studies have reported the prolonged use of membranes and the use of specific operational parameters during membrane filtration to be responsible for the emergence of predominance within different bacterial populations in biofilms (Avadhanula, 2011; Anand and Singh, 2013; Sánchez, 2018). Also, Anand and Singh (2013) reported that selective bacterial species that developed resistance to the chemical cleaning protocols over prolonged use of membrane acquired predominance in the biofilm microflora. A validation study was also conducted to establish the predominance of $B$. subtilis by natural selection using a nonselective nutrient broth. This experiment allowed the multispecies microflora to interact and demonstrate the emergence of predominance, similarly to that demonstrated in the coculture growth using combinations of 2 isolates each. The first cycle of incubation of membrane pieces in TSB yielded all 6 isolates previously identified as the constitutive microflora of the reverse osmosis membrane. As the cycles of TSB transfers and subsequent incubations progressed, the types of isolates started to decrease, and finally only 1 isolate of $B$. subtilis was enumerated at the end of the fifth transfer. Therefore, it can be inferred that B. subtilis emerged predominant within both coculture and multispecies growth conditions. Previous studies have reported that the natural selection process in a well-mixed environment accelerates the transition process by recurring life cycles (Korolev et al., 2012). A similar approach was adopted in this study where subsequent transfers were used to continue the process, which resulted in the emergence of the predominance of one isolate over the others. Also, it is important to note that there is a possibility for the isolates in the microbial suspension to coexist by simply maintaining a balance between growth rates and effective toxicity (Weber et al., 2014).

Based on the results obtained, it can be concluded that the isolates within the whey reverse osmosis biofilm microflora demonstrated the emergence of the predominance of $B$. subtilis among all the isolates under the conditions of the experiment. This study thus provides important information related to the constitutive microflora present within an 18-mo-old reverse osmosis membrane and identification of B. subtilis as the predominant species within the biofilm microflora using coculture and mixed-species growth.

\section{ACKNOWLEDGMENTS}

This work was funded by Dairy Management Inc. (Rosemont, IL) and supported by the Agricultural Experiment Station at South Dakota State University (Brookings). The authors acknowledge the Veterinary Science Department at South Dakota State University for conducting MALDI-TOF and Cornell University (Ithaca, NY) for conducting rpoB sequencing. The authors have not stated any conflicts of interest. 


\section{REFERENCES}

Abrudan, M. I., F. Smakman, A. J. Grimbergen, S. Westhoff, E. L. Miller, G. P. Van Wezel, and D. E. Rozen. 2015. Socially mediated induction and suppression of antibiosis during bacterial coexistence. Proc. Natl. Acad. Sci. USA 112:11054-11059. https://doi .org/10.1073/pnas.1504076112.

Anand, S., A. Hassan, and M. Avadhanula. 2012. The effects of biofilms formed on whey reverse osmosis membranes on the microbial quality of the concentrated product. Int. J. Dairy Technol. 65:451455. https://doi.org/10.1111/j.1471-0307.2012.00848.x.

Anand, S., and D. Singh. 2013. Resistance of the constitutive microflora of biofilms formed on whey reverse-osmosis membranes to individual cleaning steps of a typical clean-in-place protocol. J. Dairy Sci. 96:6213-6222. https://doi.org/10.3168/jds.2013-7012.

Anand, S., D. Singh, M. Avadhanula, and S. Marka. 2014. Development and control of bacterial biofilms on dairy processing membranes. Compr. Rev. Food Sci. Food Saf. 13:18-33. https://doi .org/10.1111/1541-4337.12048.

Avadhanula, M. 2011. Formation of bacterial biofilms on spiral wound reverse osmosis whey concentration membranes. MS Thesis. South Dakota State Univ., Brookings.

Borenstein, D. B., Y. Meir, J. W. Shaevitz, and N. S. Wingreen. 2013. Non-local interaction via diffusible resource prevents coexistence of cooperators and cheaters in a lattice model. PLoS One 8:e63304. https://doi.org/10.1371/journal.pone.0063304.

Brown, K. L. 2000. Control of bacterial spores. Br. Med. Bull. 56:158171. https://doi.org/10.1258/0007142001902860.

Buehner, K. P. 2014. The incidence of spores and thermoduric bacteria in midwest dairies and commercial NFDM samples and their reduction via ultrasonication. MS Thesis. South Dakota State Univ., Brookings.

Chamberland, J., G. Beaulieu-Carbonneau, M. Lessard, S. Labrie, L. Bazinet, A. Doyen, and Y. Pouliot. 2017a. Effect of membrane material chemistry and properties on biofouling susceptibility during milk and cheese whey ultrafiltration. J. Membr. Sci. 542:208-216. https://doi.org/10.1016/j.memsci.2017.08.012.

Chamberland, J., M. H. Lessard, A. Doyen, S. Labrie, and Y. Pouliot. 2017b. A sequencing approach targeting the 16S rRNA gene unravels the biofilm composition of spiral-wound membranes used in the dairy industry. Dairy Sci. Technol. 96:827-843. https://doi .org/10.1007/s13594-016-0305-2.

Chinen, K. 2017. Klebsiella pneumoniae liver abscess. J. Gen. Fam. Med. 18:466-467. https://doi.org/10.1002/jgf2.90.

Daufin, G., J. P. Escudier, H. Carrére, S. Bérot, L. Fillaudeau, and M. Decloux. 2001. Recent and emerging applications of membrane processes in the food and dairy industry. Food Bioprod. Process. 79:89-102. https://doi.org/10.1205/096030801750286131.

DeBach, P. 1966. The competitive displacement and coexistence principles. Annu. Rev. Entomol. 11:183-212. https://doi.org/10.1146/ annurev.en.11.010166.001151.

D'Souza, N. M., and A. J. Mawson. 2005. Membrane cleaning in the dairy industry: A review. Crit. Rev. Food Sci. Nutr. 45:125-134. https://doi.org/10.1080/10408690490911783.

Flint, S. H., P. J. Bremer, and J. D. Brooks. 1997. Biofilms in dairy manufacturing plant-Description, current concerns and methods of control. Biofouling 11:81-97. https://doi.org/10.1080/ 08927019709378321.

Fredrickson, A. G., and G. Stephanopoulos. 2017. Microbial competition. Science 213:972-979.

Frost, I., W. P. J. Smith, S. Mitri, A. S. Millan, Y. Davit, J. M. Osborne, J. M. Pitt-Francis, R. C. MacLean, and K. R. Foster. 2018.
Cooperation, competition and antibiotic resistance in bacterial colonies. ISME J. 12:1582-1593. https://doi.org/10.1038/s41396 -018-0090-4.

Hardy Diagnostics. 2020. Letheen Media: Instructions for Use. Hardy Diagnostics, Santa Maria, CA. Accessed Oct. 9, 2020. https://catalog.hardydiagnostics.com/cp_prod/Content/hugo/ LetheenMedia.htm.

Hassan, A. N., S. Anand, and M. Avadhanula. 2010. Microscopic observation of multispecies biofilm of various structures on whey concentration membranes. J. Dairy Sci. 93:2321-2329. https://doi .org $/ 10.3168 /$ jds.2009-2800.

Korolev, K. S., M. J. I. Müller, N. Karahan, A. W. Murray, O. Hallatschek, and D. R. Nelson. 2012. Selective sweeps in growing microbial colonies. Phys. Biol. 9:026008. https://doi.org/10.1088/ 1478-3975/9/2/026008.

Marchand, S., J. De Block, V. De Jonghe, A. Coorevits, M. Heyndrickx, and L. Herman. 2012. Biofilm formation in milk production and processing environments; influence on milk quality and safety. Compr. Rev. Food Sci. Food Saf. 11:133-147. https://doi.org/10 .1111/j.1541-4337.2011.00183.x.

Marka, S., and S. Anand. 2018. Feed substrates influence biofilm formation on reverse osmosis membranes and their cleaning efficiency. J. Dairy Sci. 101:84-95. https://doi.org/10.3168/jds.2017-13249.

Nadell, C. D., K. Drescher, and K. R. Foster. 2016. Spatial structure, cooperation and competition in biofilms. Nat. Rev. Microbiol. 14:589-600. https://doi.org/10.1038/nrmicro.2016.84.

Sánchez, O. 2018. Microbial diversity in biofilms from reverse osmosis membranes: A short review. J. Membr. Sci. 545:240-249. https:// doi.org/10.1016/j.memsci.2017.09.082.

Stephens, P. J., and B. M. Mackey. 2003. Recovery of stressed microorganisms. Prog. Ind. Microbiol. 37:25-48.

Stratton, C. W. 1983. Pseudomonas aeruginosa. Infect. Control 4:3640. https://doi.org/10.1017/s0195941700057647.

Suwarno, S. R., X. Chen, T. H. Chong, V. L. Puspitasari, D. McDougald, Y. Cohen, S. A. Rice, and A. G. Fane. 2012. The impact of flux and spacers on biofilm development on reverse osmosis membranes. J. Membr. Sci. 405-406:219-232. https://doi.org/10.1016/ j.memsci.2012.03.012.

Tang, X., S. H. Flint, J. D. Brooks, and R. J. Bennett. 2009. Factors affecting the attachment of micro-organisms isolated from ultrafiltration and reverse osmosis membranes in dairy processing plants. J. Appl. Microbiol. 107:443-451. https://doi.org/10.1111/j.1365 $-2672.2009 .04214 . x$.

Turan, M., A. Ates, and B. Inanc. 2002. Fouling of reverse osmosis and nanofiltration membranes by dairy industry effluents. Water Sci. Technol. 45:355-360. https://doi.org/10.2166/wst.2002.0446.

US FDA (US Food and Drug Administration). 1969. Bacteriological Analytical Manual. US FDA, Washington, DC.

Weber, M. F., G. Poxleitner, E. Hebisch, E. Frey, and M. Opitz. 2014. Chemical warfare and survival strategies in bacterial range expansions. J. R. Soc. Interface 11:20140172. https://doi.org/10.1098/ rsif.2014.0172.

Weiner, B., A. Posfai, and N. Wingreen. 2017. Microbial communities with spatial structure. Accessed Oct. 9, 2020. https://www.cpe .rutgers.edu/q-bio/docs/Benjamin-Weiner.pdf.

\section{ORCIDS}

Sanjeev Anand (ํ) https://orcid.org/0000-0002-7479-9965 